\title{
Aggrecanopathies highlight the need for genetic evaluation of ISS children
}

\section{Ola Nilsson ${ }^{1,2}$}

'Division of Pediatric Endocrinology \& Center for Molecular Medicine, Department of Women's and Children's

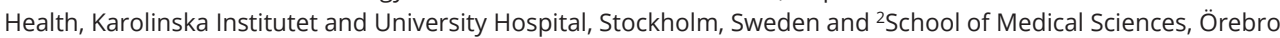
University and University Hospital, Örebro, Sweden
Correspondence

should be addressed

to O Nilsson

Email

ola.nilsson@ki.se

\begin{abstract}
Short stature is one of the most common causes for referrals to pediatric endocrinologists. However, in a majority of the children, no underlying cause can be identified and the child instead receives the unhelpful diagnosis of idiopathic short stature (ISS), often after extensive work-up and testing. Recent advances in genetic methodology have allowed for the identification of a number of different monogenic conditions within the large cohort of ISS children. Isolated short stature and advanced bone age, with or without early-onset osteoarthritis and/or osteochondritis dissecans (MIM\#165800) due to heterozygous aggrecan gene mutations exemplifies how this progress is changing the way we assess, counsel and treat children with non-endocrine growth disorders.
\end{abstract}

\section{Commentary}

In the last $15-20$ years, genome-wide approaches including genome-wide association studies, as well as exome, and genome sequencing have advanced our understanding of the genetics of human growth and made it feasible to identify genetic causes of syndromic and non-syndromic growth disorders in our short stature clinics. Taken together, the many findings have underscored several important concepts: first, that growth is a truly multigenic trait and, consequently, that the causes of short stature are spread over a large number of genes and molecular pathways; secondly, that genes important for growth for the most part are expressed in, and act directly at, the growth plates; and thirdly, that for each growth plate gene, there often is a large phenotypic spectrum ranging from severe, commonly syndromic, short stature, for example, skeletal dysplasias, to moderate isolated short stature, and even more common hypomorphic alleles and single nucleotide polymorphisms that contribute to the variation of height within the normal range (1).

One such growth plate gene, the aggrecan gene $(A C A N)$, encodes the protein aggrecan, which is the most abundant proteoglycan in hyaline cartilage and crucial to the structure and function of growth plate and articular cartilage as well as intervertebral discs of the spine. Homozygous mutations cause a severe skeletal dysplasia termed spondyloepimetaphyseal dysplasia, aggrecan type (MIM\#612813) (2), whereas heterozygous mutations result in a milder skeletal dysplasia termed spondyloepiphyseal dysplasia, Kimberley type (MIM\#608361) (3). More recently, exome/genome-wide sequencing studies have revealed that ACAN mutations also can cause isolated short stature with or without advanced bone age, with or without early-onset osteoarthritis and/or osteochondritis dissecans (MIM\#165800) (4). Additional findings in several ISS cohorts have indicated that heterozygous ACAN mutations are a fairly frequent cause of isolated short stature (5) and may explain $1-2 \%$ of all ISS (6). However, in some other short stature cohorts, ACAN mutations have been a less frequent finding (7).

In the study by Stavber et al., the authors used a set of criteria (autosomal dominant inheritance and/or advanced bone age) to select out children with the highest

Published by Bioscientifica Ltd. 
likelihood of finding a pathogenic $A C A N$ variant and they were very successful (8). They identified likely causative variants in as many as $37.5 \%$ ( 6 out of 16 families). The identified variants (two nonsense, two fameshift, one intragenic multi-exon deletion, and one missense) are all novel and co-segregated perfectly within each family (8). This is a remarkably high percentage that highlights the concepts mentioned previously and also reinforces the importance of genetic evaluation in patients/families with growth disorders of a likely monogenic etiology. The unusually high frequency of $A C A N$ mutations identified in this study are most likely due to the used inclusion criteria and maybe combined with the limited size of the cohort.

It is important to note that the phenotypes of the mutation-positive individuals reported by Stavber et al. (8) are consistent with previous reports and underscores that ACAN mutations have some features that, even if non-specific, may provide diagnostic clues, including advanced bone age, early growth cessation, osteochondritis dissecans, early-onset osteoarthritis, early-onset intervertebral disc disease, midface hypoplasia, and brachydactyly. Another interesting feature of $A C A N$-mutation-positive individuals is that they tend to have relatively long arms (5), indicating that non-weight bearing growth plates are relatively less affected than growth plates that have to support the body weight. However, all of these features are variable and not present in all patients/families.

The findings of Stavber et al. (8) also highlight the benefit of identifying a genetic diagnosis in children with short stature. In children and families with $A C A N$ mutations, it is especially important to monitor for and counsel patients and families about the increased risk of early-onset osteoarthritis and intervertebral disc disease and to promote a lifestyle that prevents and minimizes future disability and pain caused by these unfortunate features of the condition. Finally, with this condition now established as one of the more common monogenic conditions in the large group of ISS children, the next challenge will be to understand the underlying cellular and molecular mechanisms by which $A C A N$ mutations cause growth failure, accelerated skeletal maturation, early-onset osteoarthritis, and degenerative intervertebral disc disease so that novel treatment strategies that directly target the pathogenic mechanisms can be developed.
Declaration of interest

O N has received speakers' honoraria from Pfizer, Lilly, Abbott, and Biomarin, consulting fees from Ascendis and KyowaKirin, and research support from KyowaKirin and the Novo Nordisk Foundation.

\section{Funding}

The work by O N was supported by grants from the Swedish Research Council (project K2015-54X-22 736-01-4 and 2015-02227), the Swedish Governmental Agency for Innovation Systems (Vinnova) (2014-01438), Marianne and Marcus Wallenberg Foundation, IngaBritt och Arne Lundbergs forskningsstiftelse, Byggmästare Olle Engkvist Stiftelse, the Swedish Society of Medicine, Erik och Edith Fernström Foundation for Medical Research, Nyckelfonden, Stiftelsen Frimurare Barnhuset i Stockholm, the Stockholm County Council, Karolinska Institutet, Stockholm, Sweden, and Örebro University, Örebro, Sweden.

\section{References}

1 Baron J, Savendahl L, De Luca F, Dauber A, Phillip M, Wit JM \& Nilsson O. Short and tall stature: a new paradigm emerges. Nature Reviews Endocrinology 201511 735-746. (https://doi.org/10.1038/ nrendo.2015.165)

2 Tompson SW, Merriman B, Funari VA, Fresquet M, Lachman RS, Rimoin DL, Nelson SF, Briggs MD, Cohn DH \& Krakow D. A recessive skeletal dysplasia, SEMD aggrecan type, results from a missense mutation affecting the C-type lectin domain of aggrecan. American Journal of Human Genetics 200984 72-79. (https://doi.org/10.1016/j. ajhg.2008.12.001)

3 Gleghorn L, Ramesar R, Beighton P \& Wallis G. A mutation in the variable repeat region of the aggrecan gene (AGC1) causes a form of spondyloepiphyseal dysplasia associated with severe, premature osteoarthritis. American Journal of Human Genetics 200577 484-490. (https://doi.org/10.1086/444401)

4 Nilsson O, Guo MH, Dunbar N, Popovic J, Flynn D, Jacobsen C, Lui JC, Hirschhorn JN, Baron J \& Dauber A. Short stature, accelerated bone maturation, and early growth cessation due to heterozygous aggrecan mutations. Journal of Clinical Endocrinology and Metabolism 201499 E1510-E1518. (https://doi.org/10.1210/jc.2014-1332)

5 Gkourogianni A, Andrew M, Tyzinski L, Crocker M, Douglas J, Dunbar N, Fairchild J, Funari MF, Heath KE, Jorge AA et al. Clinical characterization of patients with autosomal dominant short stature due to aggrecan mutations. Journal of Clinical Endocrinology and Metabolism 2017102 460-469. (https://doi.org/10.1210/jc.2016-3313)

6 Hauer NN, Sticht H, Boppudi S, Buttner C, Kraus C, Trautmann U, Zenker M, Zweier C, Wiesener A, Jamra RA et al. Genetic screening confirms heterozygous mutations in ACAN as a major cause of idiopathic short stature. Scientific Reports 20177 12225. (https://doi. org/10.1038/s41598-017-12465-6)

7 Freire BL, Homma TK, Funari MFA, Lerario AM, Vasques GA, Malaquias AC, Arnhold IJP \& Jorge AAL. Multigene sequencing analysis of children born small for gestational age with isolated short stature. Journal of Clinical Endocrinology and Metabolism 2019104 2023-2030. (https://doi.org/10.1210/jc.2018-01971)

8 Stavber L, Hovnik T, Kotnik P, Lovrečić L, Kovač J, Tesovnik T, Bertok S, Dovč K, Debeljak M, Battelino T, et al. High frequency of pathogenic ACAN variants including an intragenic deletion in selected individuals with short stature. European Journal of Endocrinology 2020182 243-253. (https://doi.org/10.1530/EJE-19-0771) 